\title{
Duty Cycle of Radio Mode Feedback
}

\author{
Laura Bîrzan \\ (Leiden Observatory)
}

Collaborators: David Rafferty, Huub Röttgering (Leiden Obs.),

Brian McNamara (U. Waterloo), Paul Nulsen (CfA),

Michael Wise (ASTRON) 


\section{Balancing Cooling with Cavities}

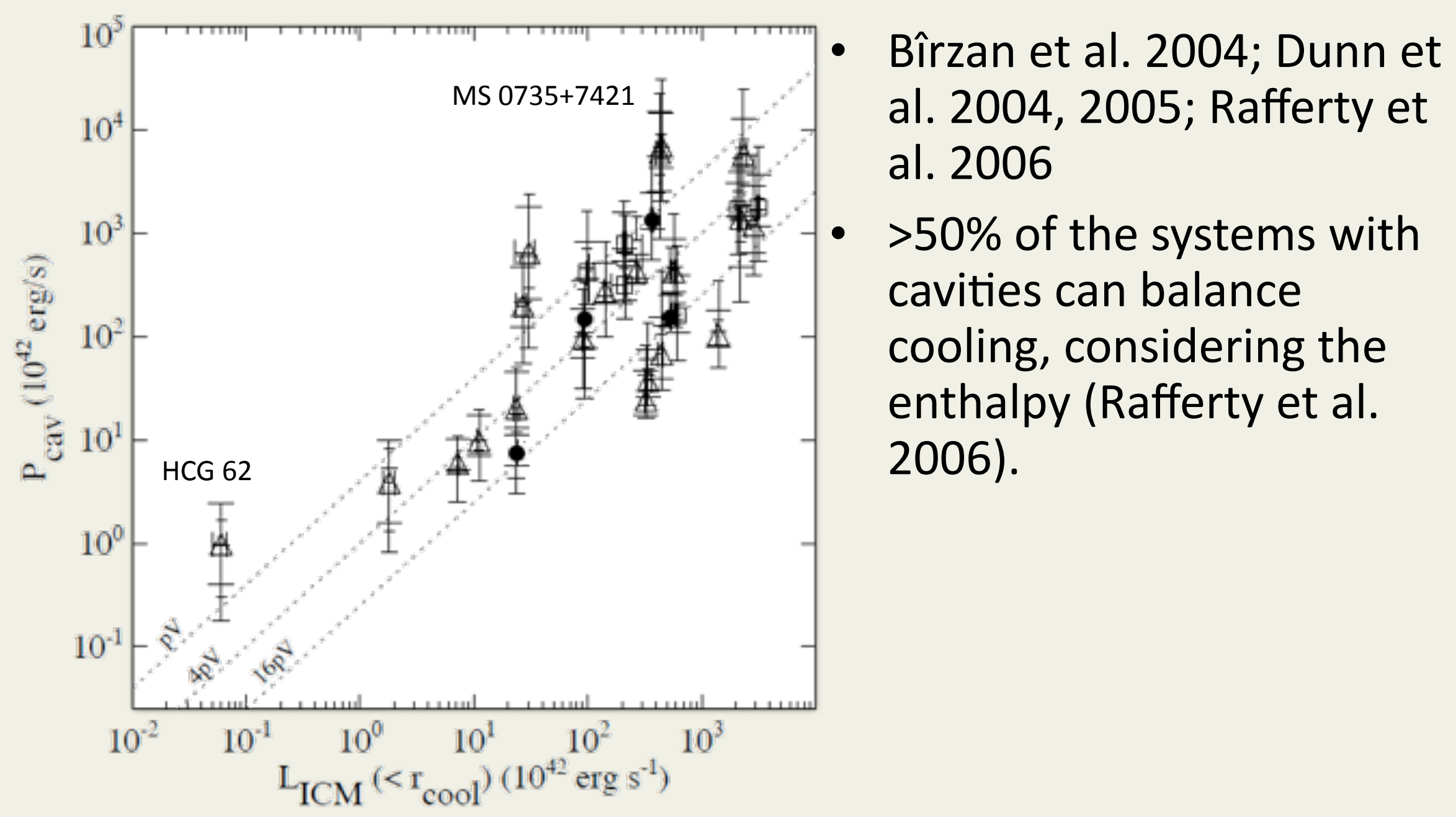




\section{Balancing Cooling with Cavities}

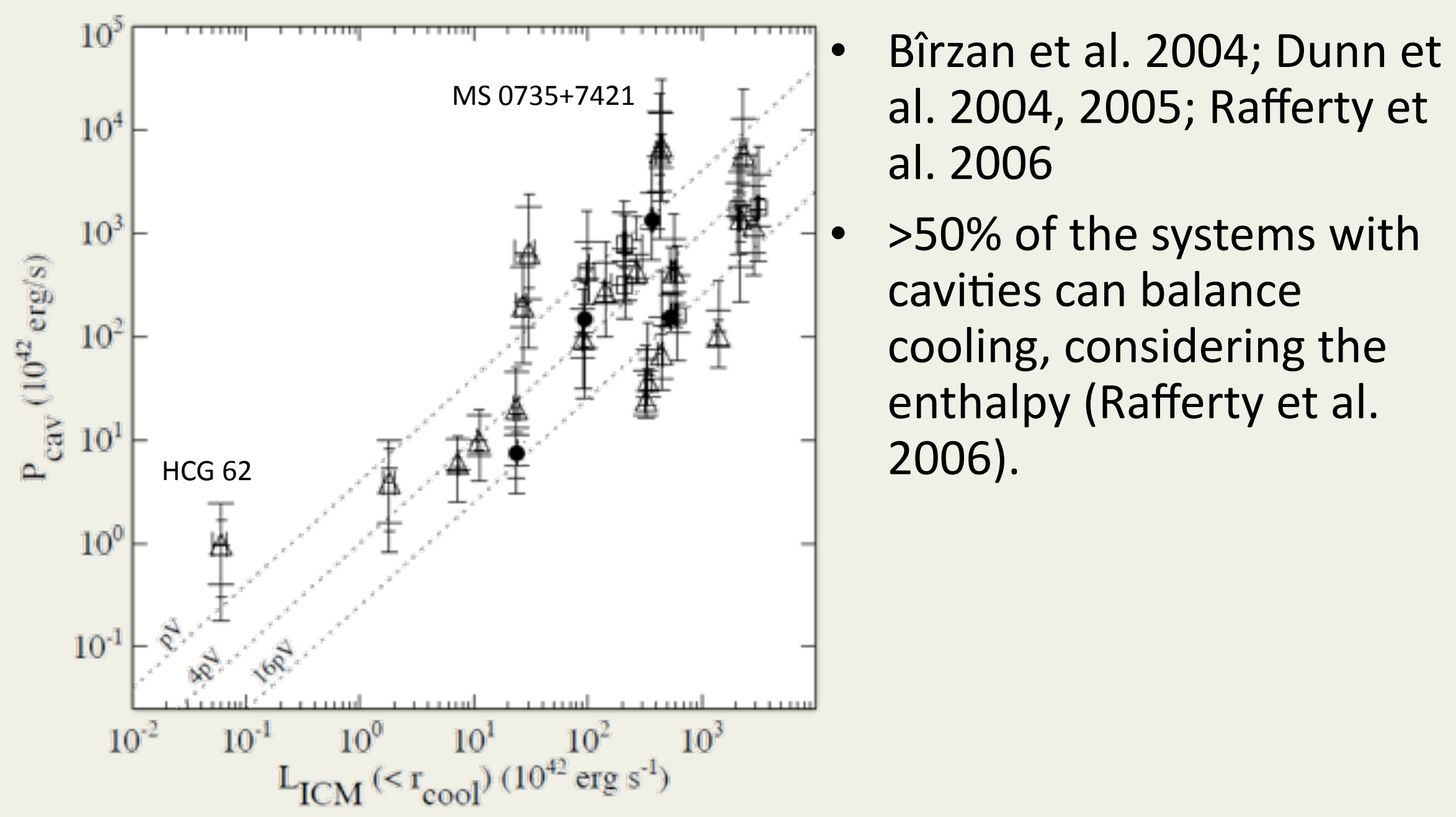




\section{Cooling Flow Clusters}

- We do not find cavities in all cooling flow clusters

- Some clusters may not have cavities (e.g., A1650, Donahue et al. 2005):

- Heating from "sloshing" (Zuhone et al. 2010)

- In a cooling stage (e.g., A1068, McNamara et al. 2004)

- Location and orientation (Ensslin \& Heinz 2002; Bruggen et al. 2009)

- Depth of the observation

- Goal: To understand the biases/selection effects in the detectability of current X-ray cavity samples

$\Rightarrow$ Need complete samples of cooling flows 


\section{Subsamples of Cooling Flow Clusters}

- Complete samples (flux limited): B55 and HIFLUGCS

- Identify cooling flow systems (Chen et al. 2007, Sanderson et al. 2006, 2009; Cavagnolo et al. 2009, etc.)

- Underling origin for the CF/NCF dichotomy:

- Separation occurs early on in the cluster evolution (pre-heating; e.g., McCarthy et al. 2008):

- Heating from mergers (Poole et al. 2008, Burns et al. 2008),

- Heating from TeV gamma rays from blazars (Pfrommer et al. 2011)

- Separation occurs late:

- Destruction of CF due to merger (Rossetti \& Molendi 2010, 2011)

- Destruction due to powerful AGN (Guo \& Oh 2009)

- Both are improbable? (Poole et al. 2008, Pfrommer et al. 2011) 


\section{CF/NCF Separation (continued)}

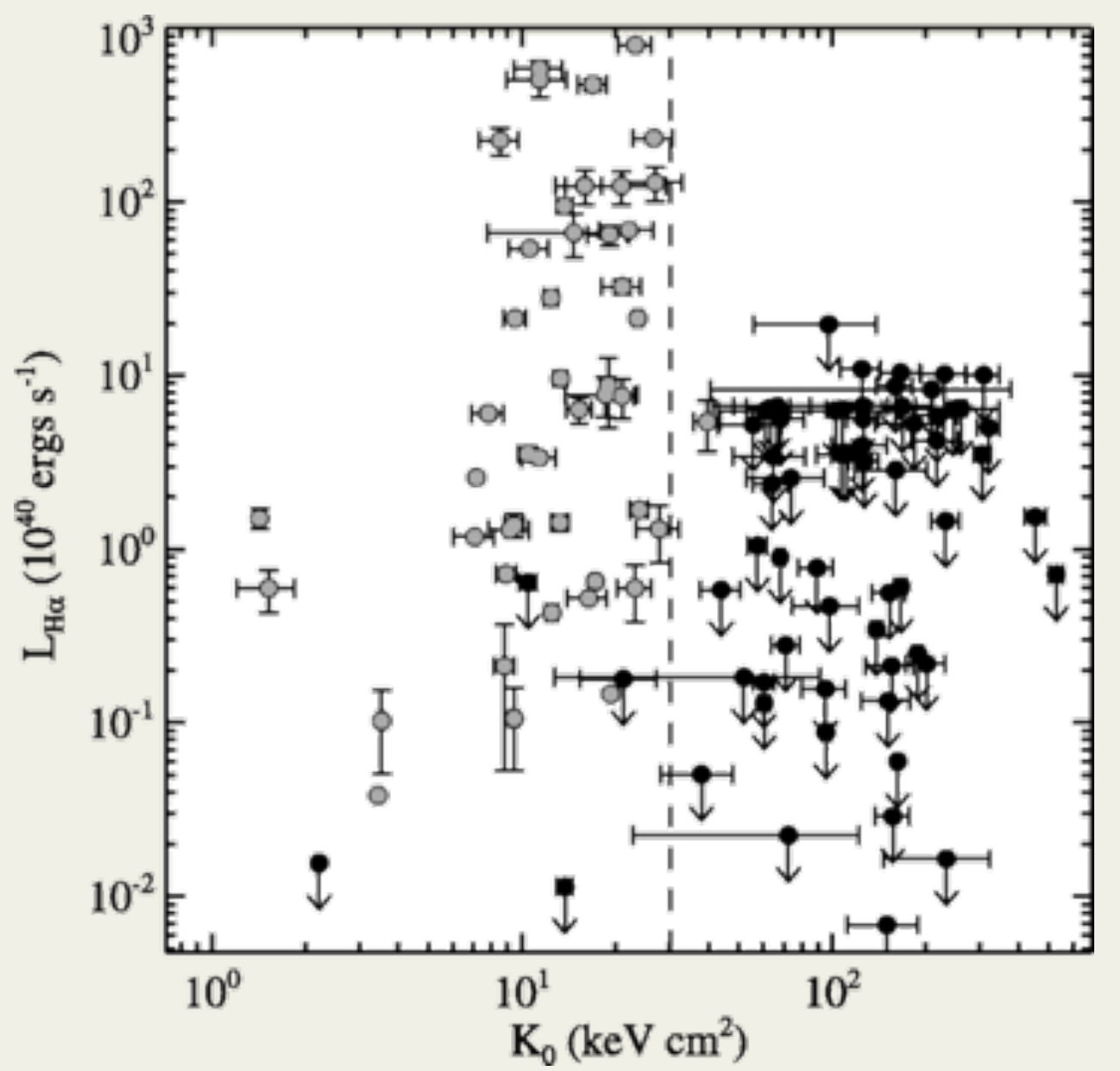

Cavagnolo et al. (2008)

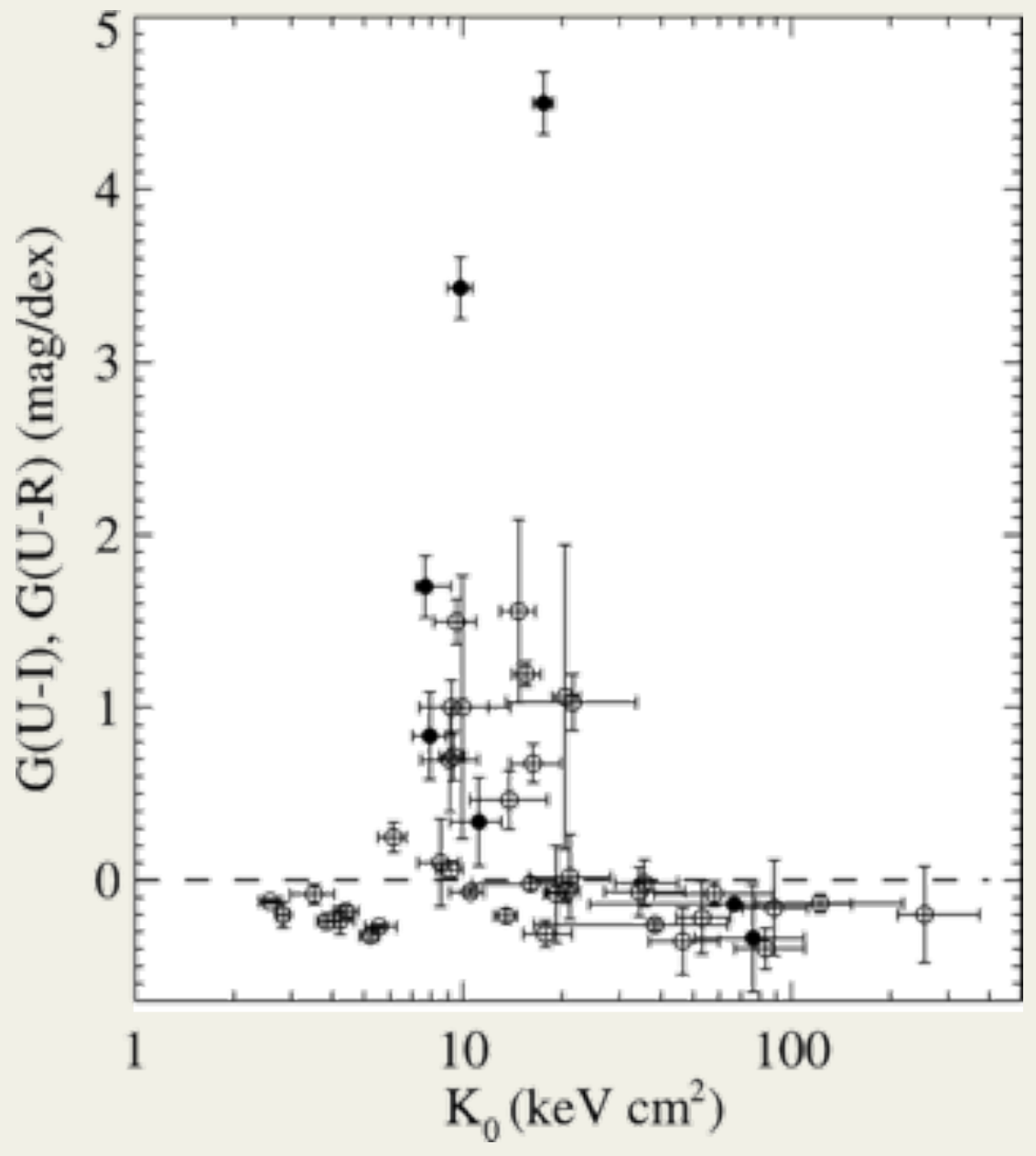

Rafferty et al. (2008) 


\section{CF/NCF Separation (continued)}

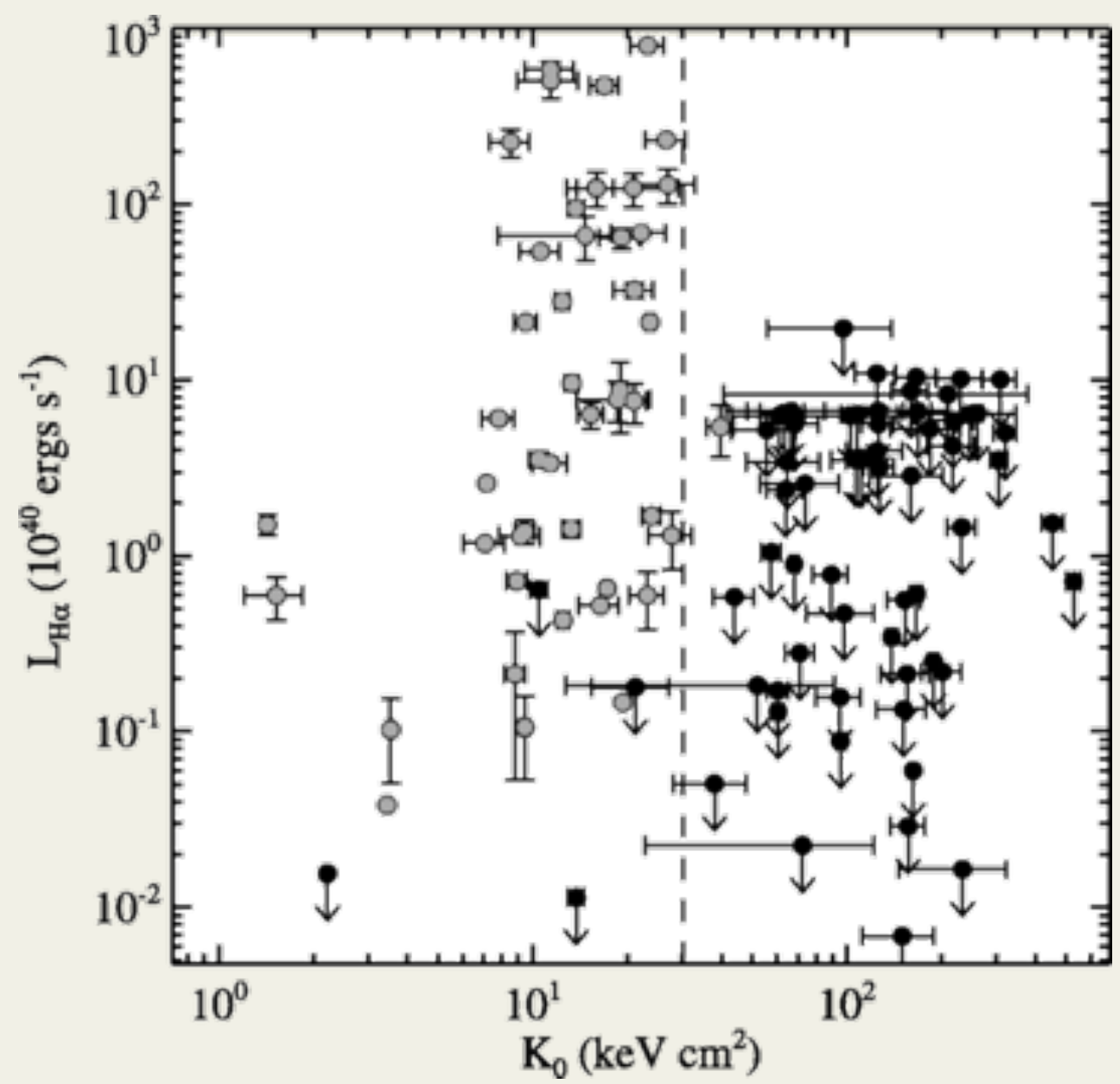

Cavagnolo et al. (2008)

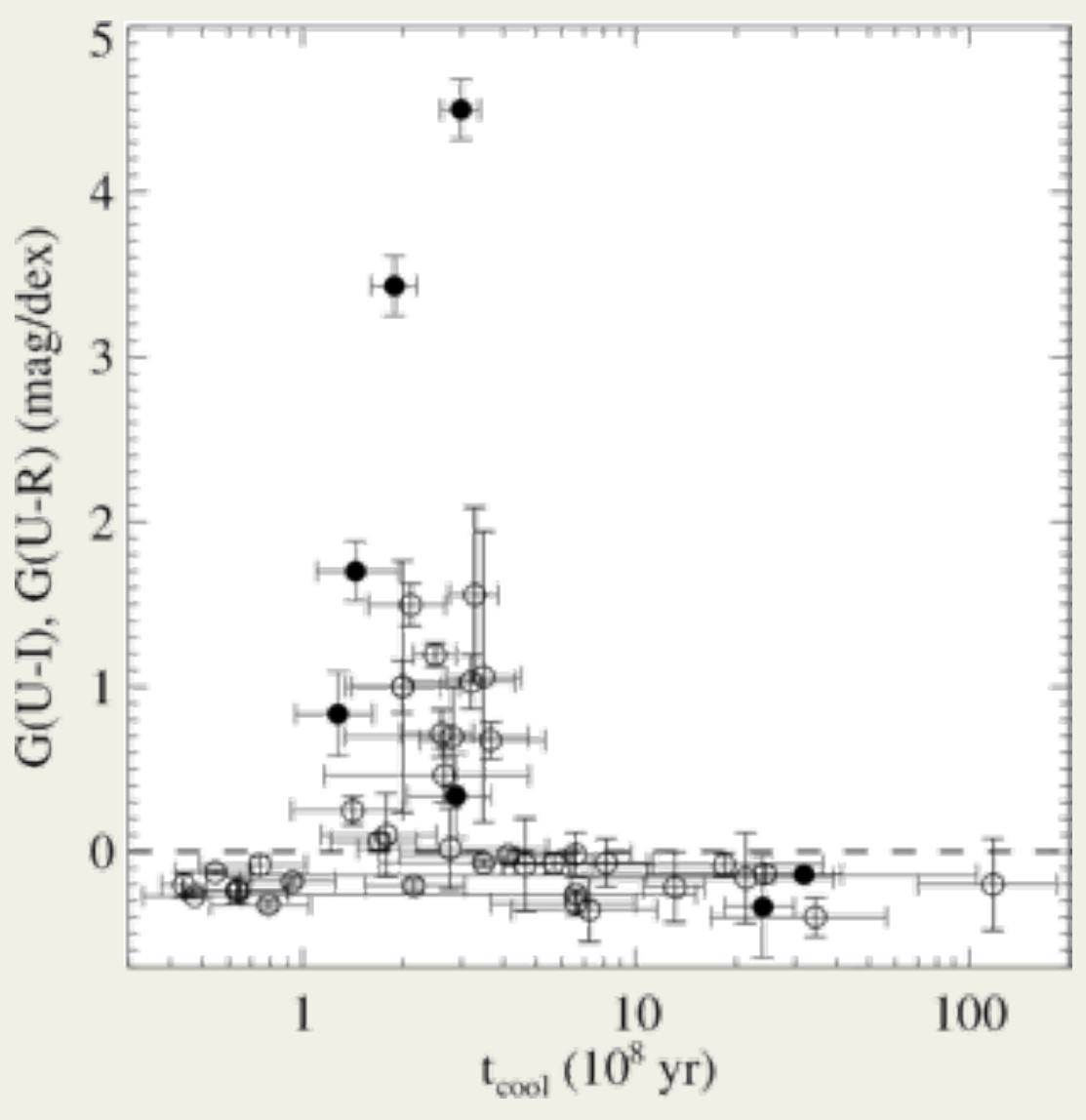

Rafferty et al. (2008) 


\section{CF/NCF Separation (continued)}

- Star formation and $\mathrm{H}-\alpha$ (and hence cooling) seems to occur if

$\eta_{\text {min }}=\min \left(\frac{\kappa T}{\Lambda n_{\mathrm{e}} n_{\mathrm{H}} r^{2}}\right) \sim \frac{1}{f_{\mathrm{c}}} \leq 5$

(Voit et al. 2008)

- Similar to Sharma et al. (2011) criterion:

$$
\min \left(t_{\text {cool }} / t_{\text {ff }}\right) \lesssim 10
$$

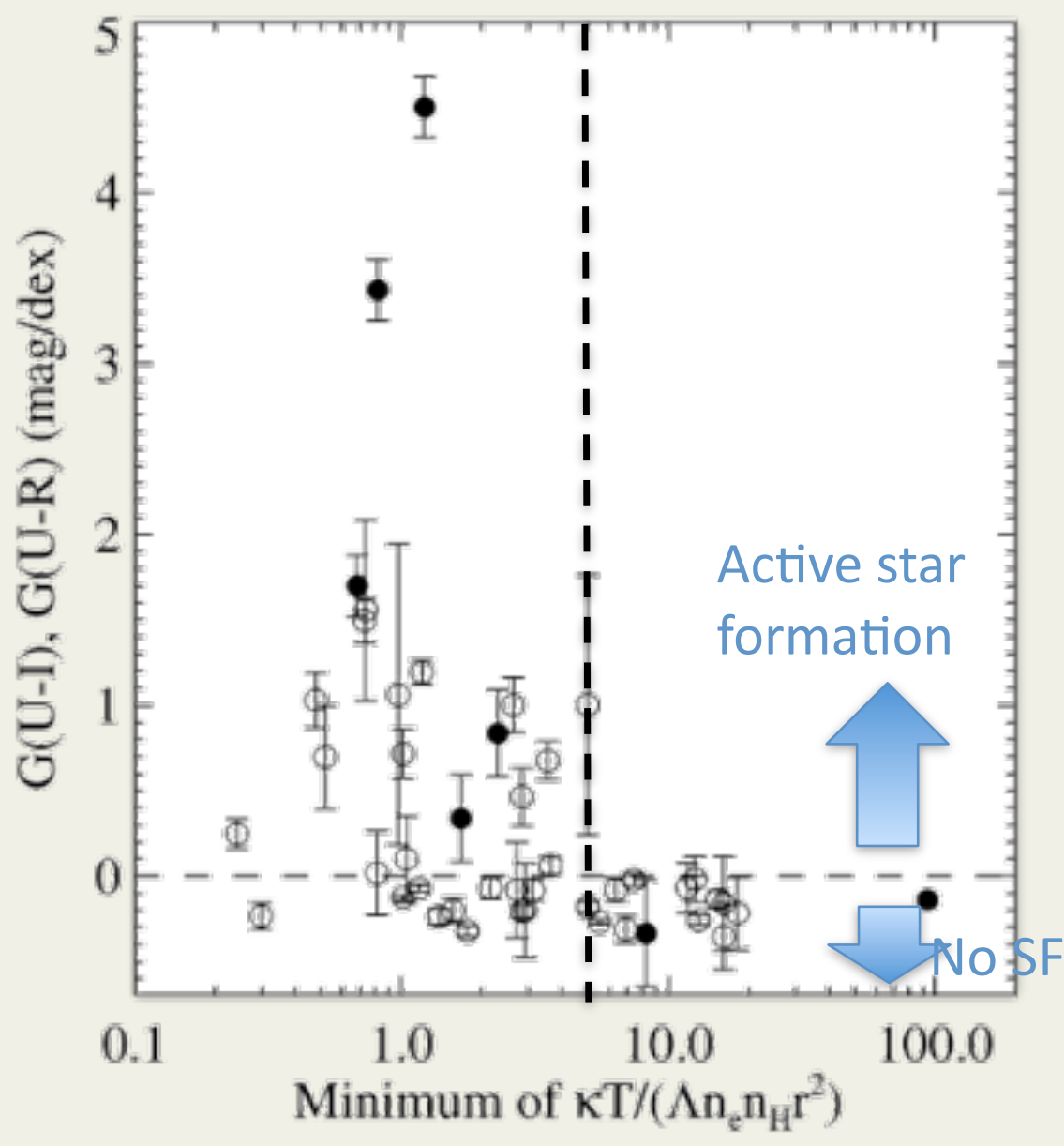

Voit et al. (2008) 


\section{CF vs NCF}

- CF systems also separate based on radio luminosity of BCGs (from Mittal et al. 2009):

- All high-radio-luminosity systems are consistent with $t_{\text {cool }}$ $\leqslant 5 \times 10^{8} \mathrm{yr}$

- And projected optical--Xray separation

- Mergers are randomly distributed:

$\Rightarrow$ mergers are not primary means of quenching cooling

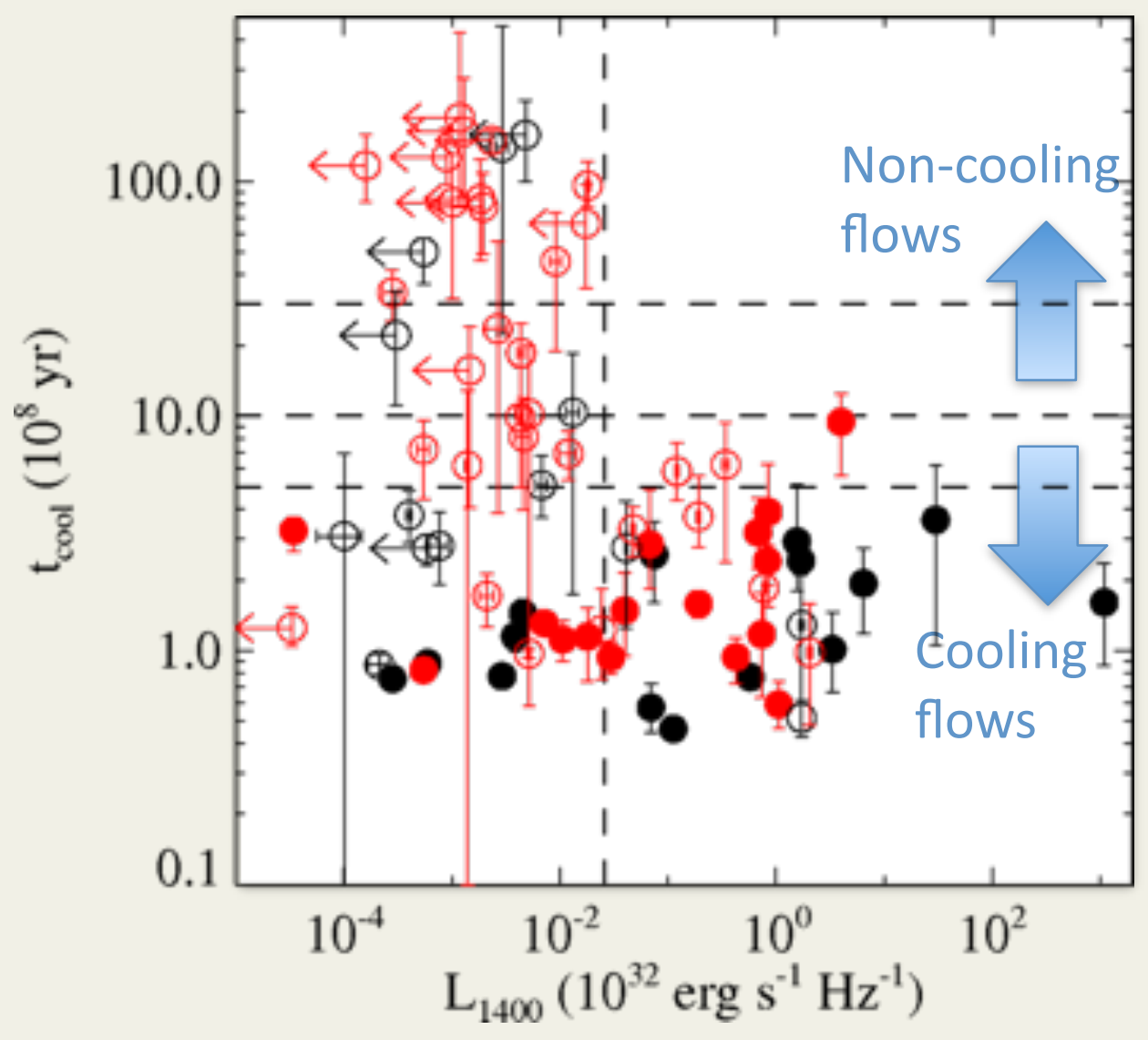

Bîrzan et al. (2012 in prep) 


\section{CF vs NCF}

- CF systems also separate based on radio luminosity of BCGs (from Mittal et al. 2009):

- All high-radio-luminosity systems are consistent with $t_{\text {cool }}$ $\leqslant 5 \times 10^{8} \mathrm{yr}$

- And projected optical--Xray separation

- Mergers are randomly distributed:

$\Rightarrow$ mergers are not primary means of quenching cooling

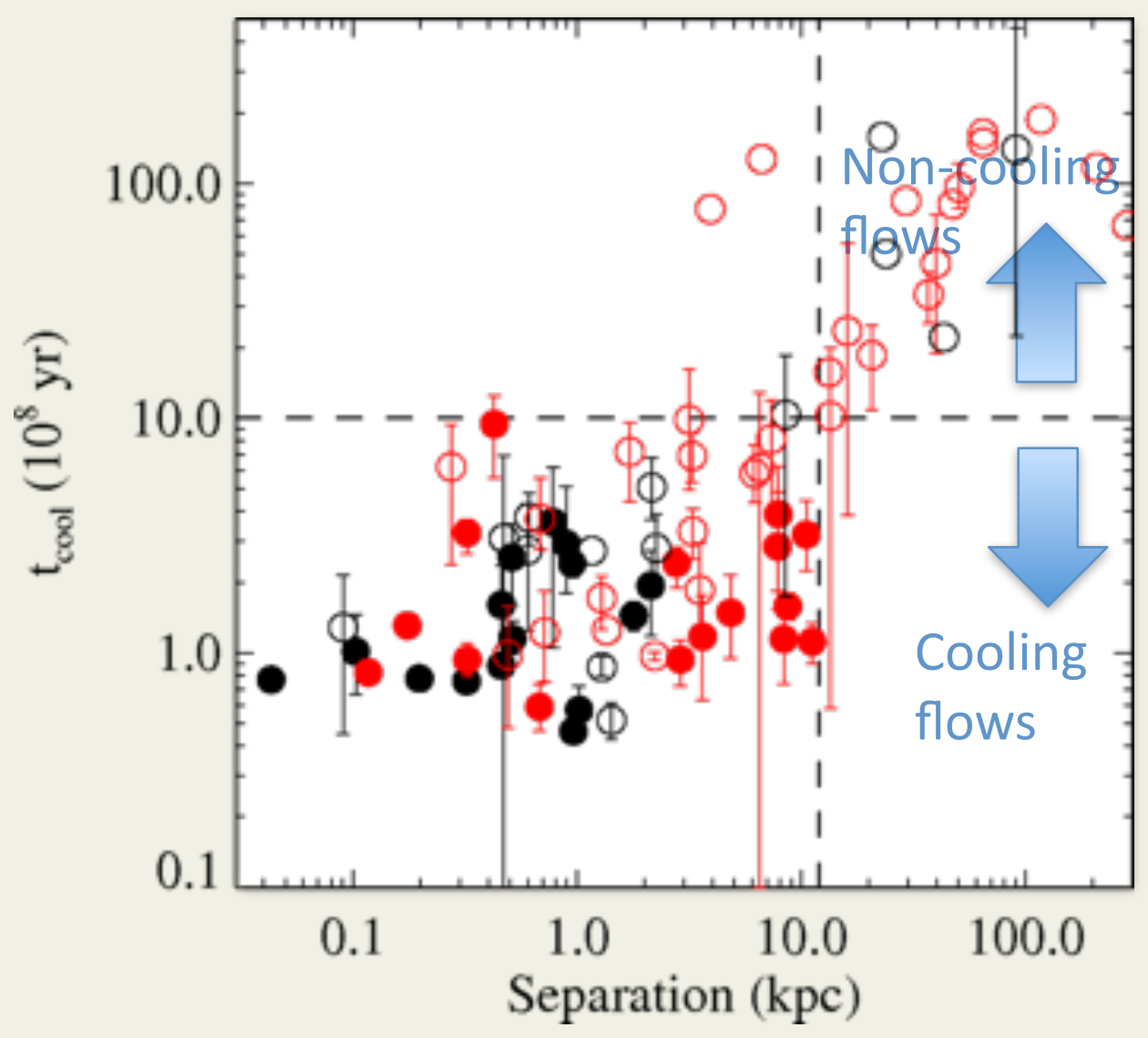

Bîrzan et al. (2012 in prep) 


\section{Cooling Flow Samples}

- $\eta_{\text {mirøf } 5 \approx \text { Central cooling }}$ time of $5 \times 10^{8} \mathrm{yr}$ (only 1 object is different: A2065)

- 49 systems require heating: 31 have detected cavities

- For 18 systems without detected cavities, we perform simulations to place limits on how much energy may be present but undetected

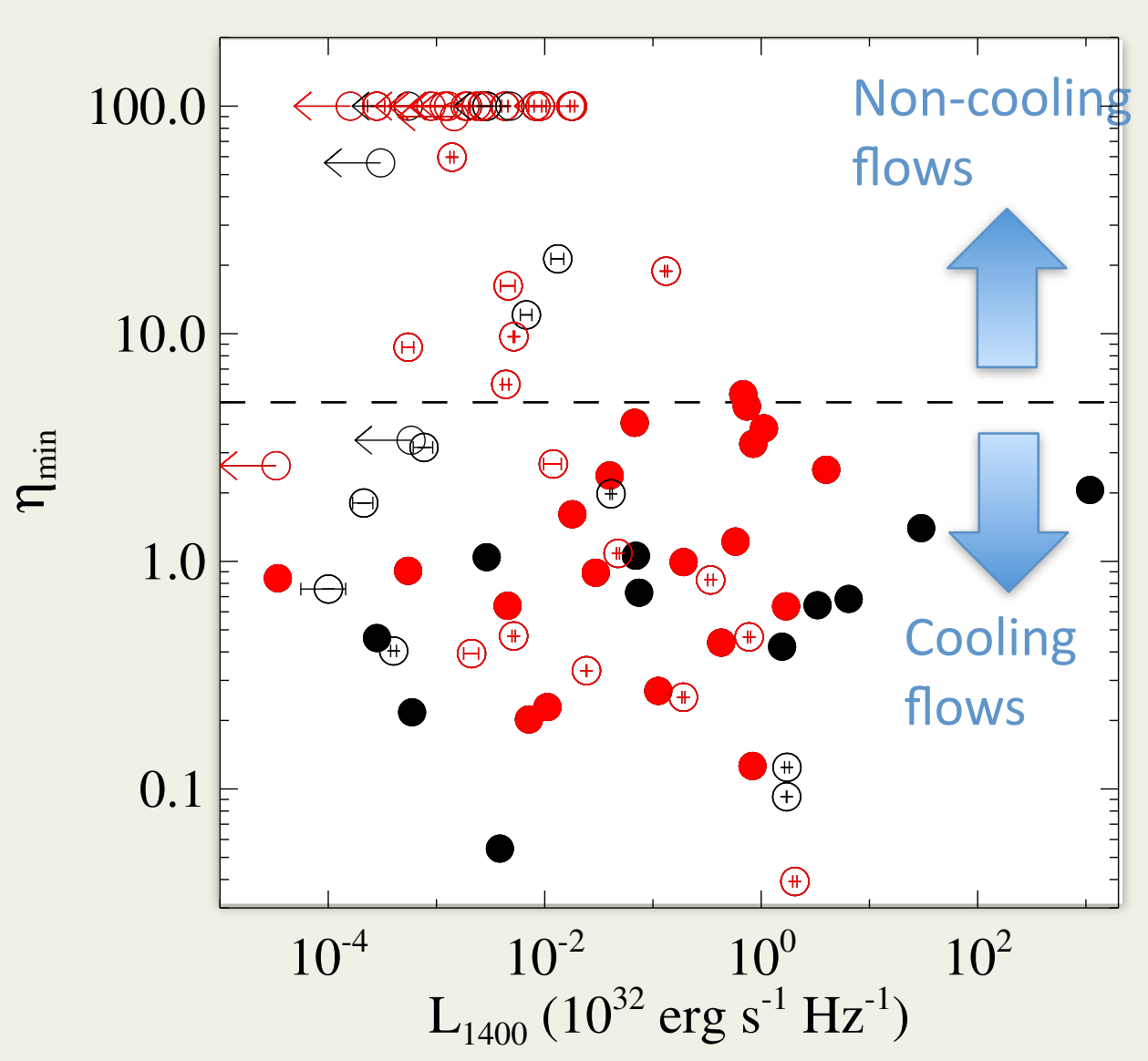




\section{Simulation Parameters}

- Double- $\beta$ model $>$ 3D emissivity $>2 \mathrm{D}$ SB $>$ MARX > Sim. image

- Assuming adiabatic expansion (to place limits on bubble sizes and locations) and buoyancy velocity (to calculate ages)

- Calculate the injection radius assuming:

$$
4 p V \sim P_{\text {cav }} t \sim L_{\mathrm{X}} t
$$

where $t$ is the time between the outbursts $\left(\approx 10^{8}\right.$ yr) 


\section{Heating vs. Cooling for Complete}

Bîrzan et al. (2012 in prep)

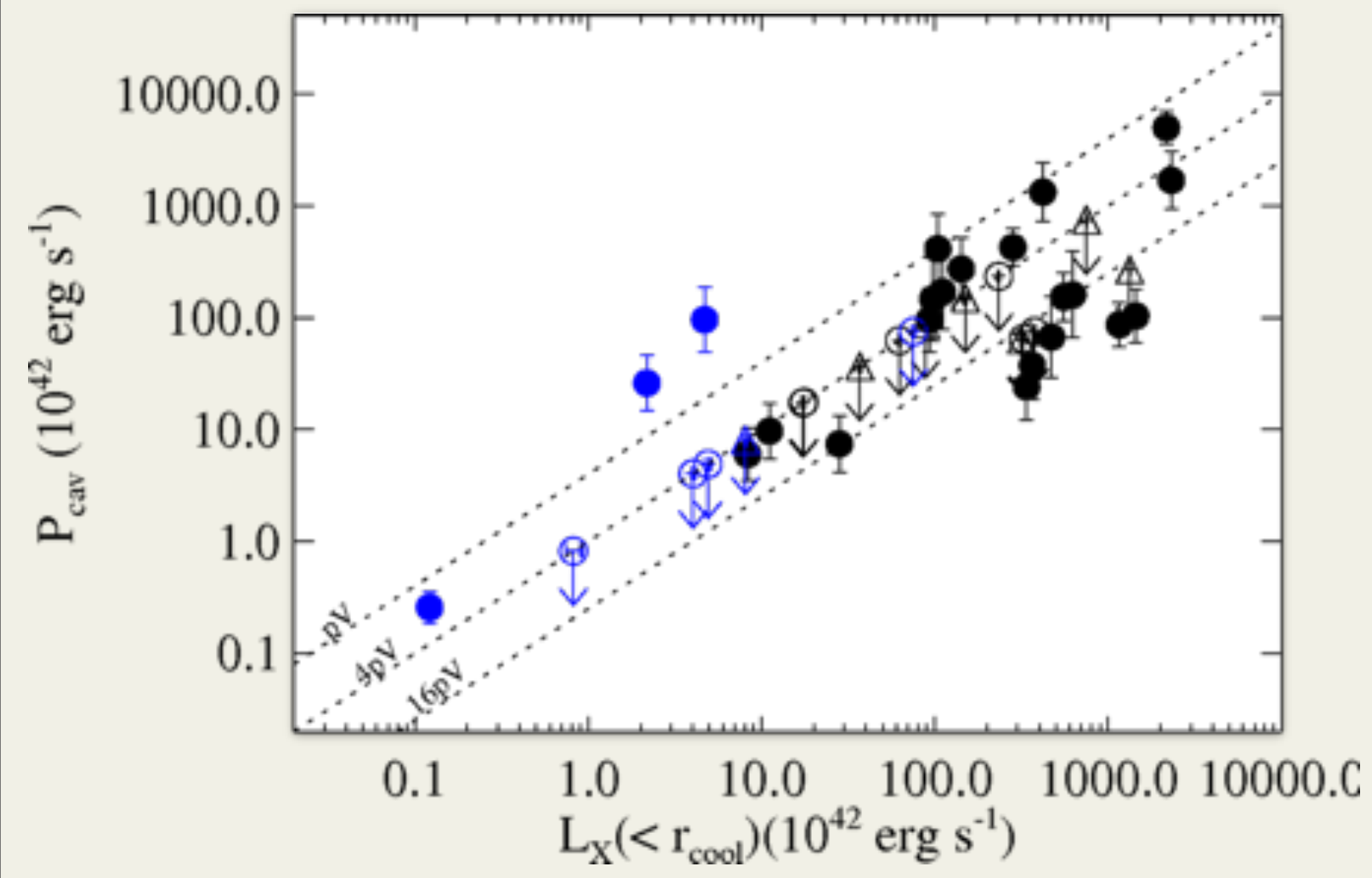

- Roughly $\sim 65 \%$ of cooling flow clusters have detected cavities ( $80 \%$ when we exclude the corona class)

- Similar to the Dunn et al. (2006) finding for a sample of cooling flows ( 70\%)

Corona class systems (Sun et al. 2007, 2009)

- Cooling flows with detected bubbles

$\downarrow$ Cooling flows without detected bubbles 


\section{Heating vs. Cooling for Complete}

Bîrzan et al. (2012 in prep)

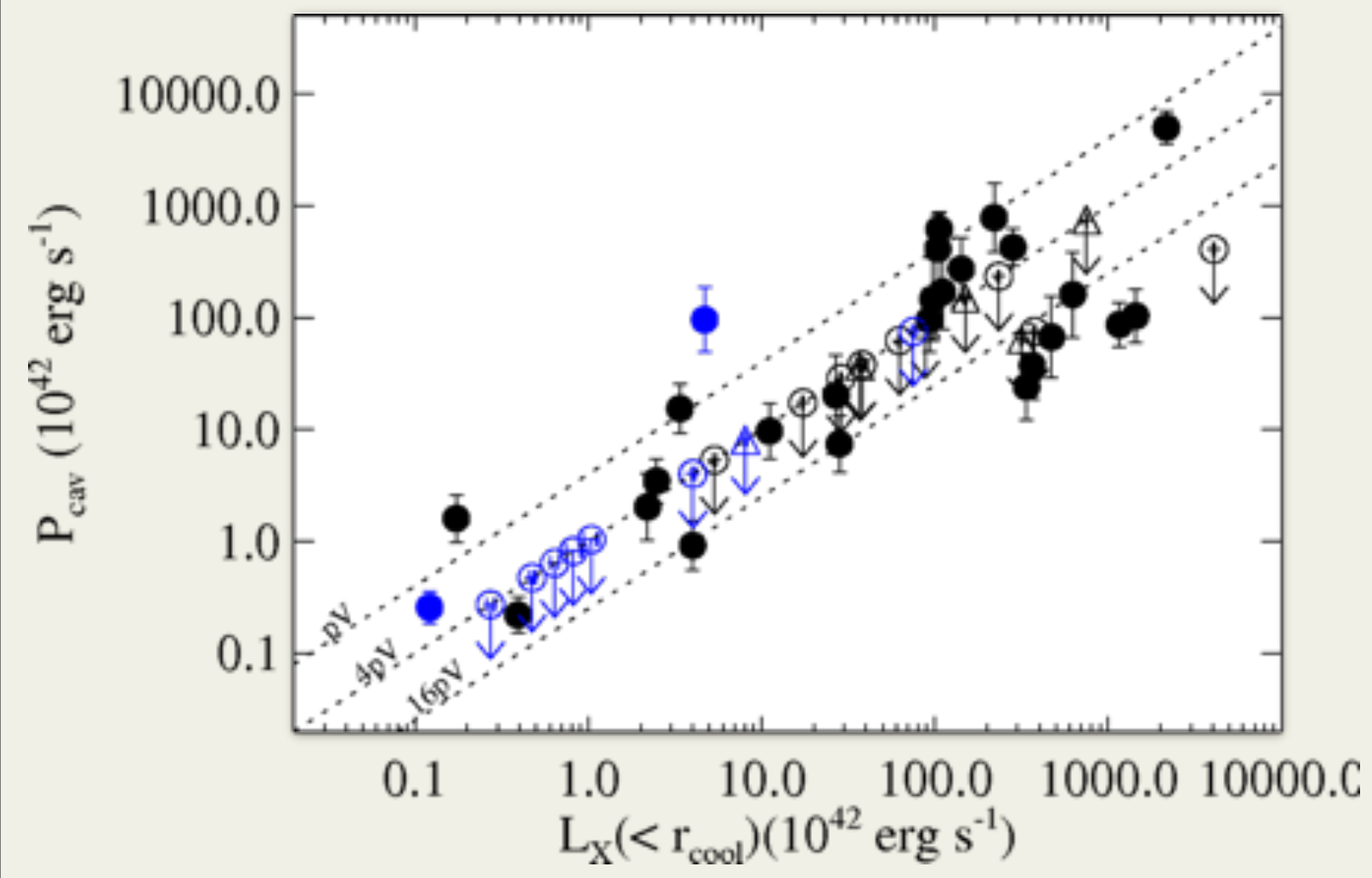

- Roughly $\sim 65 \%$ of cooling flow clusters have detected cavities ( $80 \%$ when we exclude the corona class)

- Similar to the Dunn et al. (2006) finding for a sample of cooling flows ( 70\%)

Corona class systems (Sun et al. 2007, 2009)

- Cooling flows with detected bubbles

$\downarrow$ Cooling flows without detected bubbles 


\section{Summary and Future Work}

- Roughly $65 \%$ of cooling flows ( $40 \%$ of all clusters) have detected cavities

- Of the others, most could have significant cavity power yet remain undetected in existing images (under simple assumptions), or may be in a cooling stage

- Further work needed:

- Investigate with radio images (Burns et al 1990, Mittal et al. 2009) using EVLA, GMRT, LOFAR

- Add rims to cavities

- Investigate different schemes for bubble evolution 


\section{What can LOFAR add?}

- For NCF: search for radio halos (see Cassano talk)

- study complete samples to understand the fraction of NCF systems with halos

- However, some CF systems might also have a halo (e.g., A1689, Vacca et al. 2011)

- For CF: mini-haloes, re-energizing of the old AGN activity by sloshing 